\title{
Conception Et Analyse De La Structure Factorielle D'un Outil Informatisé D'évaluation Des Enseignements
}

\author{
Chemsi Ghizlane \\ Sadiq Mounir \\ Radid Mohamed \\ Talbi Mohammed
}

Observatoire De Recherches En Didactique Et Pédagogie Universitaire

(ORDIPU). Faculté des Sciences Ben M'Sik, Hassan II University of Casablanca, Maroc.

doi: 10.19044/esj.2016.v12n7p109 URL:http://dx.doi.org/10.19044/esj.2016.v12n7p109

\begin{abstract}
Students' assassment of the quality of teaching and educationis a valuable tool through which lecturers can find out what the participants think of their educational task. This is a kind of assessment which is embeded right in the centre of the pedagogical act and which brings together the principal agents pertaining to the university teaching system (students, lecturers, and the staff in charge). However, this form of assessment is disputed as it encounters significant obstacles, namely difficulties in terms of the management of this assessment as well as in terms of reliability and validity of its measuring tools. This paper aims at providing the necessary steps for an elaboration and a foundation of an online measure of evaluating teaching and education. Such a computerised evaluation is to be administeredby students who are studying at the Faculty of Science, Ben M'Sik - Casablanca, Morocco. To begin with, in this online tool of evaluatin we shall describe the stages of this elaboration, includingcertain chosen criteria. Secondly, we will show the way in which the functionalities and possibilities of an online tool of assessment can effectively be handledby students within an institute of Higher Education. In concludion,we end this study by providing a factor analysis of this computerised instrument: this is to be carried out through the most important measures, namely, validity and authenticity.
\end{abstract}


Keywords: Computerised measuring instrument, Evaluation of teaching by students, Evaluating teaching and education, Validity, Fidelity, factor analysis

\section{Résumé}

L'évaluation des enseignements et des formations par les étudiants (EEFE) est un outil mis à la disposition des enseignants pour recueillir l'avis des étudiants sur la qualité des enseignements et des formations. C'est une forme d'évaluation qui est au cœur de l'acte pédagogique et se situe à la confluence des principaux acteurs du système d'enseignement universitaire (les étudiants, les enseignants et les responsables). Pourtant, cette pratique reste très discutée et rencontre d'importants problèmes notamment les problèmes de gestion de cette évaluation ainsi le problème de fidélité et de validité de ces instruments de mesure. L'objectif de cette étude est de présenter les démarches d'élaboration et de validation d'un outil d'évaluation des enseignements et des formations informatisé par les étudiants au sein de la faculté des sciences Ben M'sik-Casablanca- Maroc. Dans un premier temps nous y décrirons les étapes d'élaboration de l'outil et les critères choisis. Ensuite nous présentons ses fonctionnalités et ses possibilités pour gérer efficacement une démarche d'évaluation des enseignements par les étudiants dans un établissement d'enseignement supérieur. Enfin, nous conclurons par la structure factorielle de cet instrument, soit les principales mesures de validité et de fidélité.

Mots clés: Instrument de mesure informatisé, évaluation des enseignements par les étudiants, évaluation des formations, Validité, Fidélité, Analyse Factorielle

\section{Introduction}

La mondialisation suppose que le système universitaire est compétitif non seulement au niveau des performances, mais aussi au niveau de l'attraction et de la rétention des compétences. L'enseignement supérieur et la recherche scientifique sont des composants essentiels du développement culturel et socio-économique, des communautés et des nations, il est constamment confronté à des défis et devrait se transformer et se rénover pour contribuer au développement durable et à l'amélioration de la société dans son ensemble.

Pour cela, l'université devrait promouvoir, en son sein, les mécanismes capables d'assurer l'interface nécessaire avec son environnement socio-économique et fournir aux différents secteurs socioéconomiques du pays les ressources humaines nécessaires en terme de nombre, de compétence et de qualité de formation. 
Cela suppose aussi une nouvelle approche des cursus universitaires, approche qui privilègie une multidisciplinarité ainsi que le développement des ressources humaines. C'est dans ce sens que l'université marocaine a subi des remaniements dans le cadre des réformes visant à répondre au mieux aux aspirations nationales et aux mutations socioéconomiques et culturelles de la société actuelle. Cette dernière évolue de paire avec la complexité de l'acte "enseigner". La qualité de l'enseignement supérieur devient de plus en plus une exigence accrue de la part des différents acteurs concernés par les résultats de la formation universitaire, à savoir les responsables du secteur, les étudiants et la société dans son ensemble.

D'où l'importance croissante du concept "d'évaluation". C’est une composante essentielle du système éducatif depuis longtemps, elle contribue à l'amélioration continue de l'enseignement. Les tendances soulevées plus haut à propos de l'enseignement supérieur, l’importance des moyens sollicités par l'enseignement et la recherche ont été, entre autres, à l'origine de l'émergence de l'évaluation des enseignements et des formations en général. Celle-ci est plus que jamais un processus stratégique destiné à établir des diagnostics, à fonder des décisions de régulation et de réorientation des actions de formation (De Ketele \& Rogiers, 1993 ; Rege Colet, 2005b).

L'évaluation des enseignements est un outil mis à la disposition des enseignants pour recueillir l'avis des étudiants sur la qualité des enseignements. C'est aussi Selon Younes (2006), une forme d'évaluation particulièrement significative puisqu'elle touche au cœur de l'acte pédagogique et qu'elle se situe à la confluence des principaux acteurs du système d'enseignement universitaire (les étudiants, les enseignants et les responsables).

\section{Description de la problématique}

$\mathrm{Au}$ Maroc, les orientations retenues dans la loi 01-00 de l'organisation de l'enseignement supérieur (Articles $77^{3}-78^{4}-79^{5}$ ) mettent

\footnotetext{
${ }^{3}$ Loi 01-00 Article 77: Le système de l'enseignement supérieur est soumis, dans sa globalité, à une évaluation régulière, portant sur sa rentabilité interne et externe, et touchant tous les aspects pédagogiques, administratifs et de recherche. Cette évaluation se basera, en plus des audits pédagogiques, financiers et administratifs, sur l'auto évaluation de chaque établissement d'éducation et de formation, et de sondage périodique des avis des acteurs éducatifs et de leurs partenaires, dans les milieux du travail, de la science de la culture et des arts.

${ }^{4}$ Loi 01-00 Article 78 : Les établissements d'enseignement supérieur publics et privés mettent en place un système d'auto évaluation.
} 
l'accent sur la nécessité de développer une évaluation régulière du système d'enseignement supérieur dans sa globalité, notamment avec la mise en place des procédures d'évaluation afin d'accompagner la mise en place de la réforme. Cette évaluation peut prendre plusieurs formes et impliquer les acteurs du système (enseignants, étudiants, personnel administratif).

Cependant, la culture de l'évaluation du système éducatif au Maroc est encore peu développée, du fait que:

> Cette évaluation se réalise la plupart du temps sans une explicitation préalable des critères sur lesquels elle se fonde (Romainville, 2009);

Les difficultés méthodologiques et pratiques, la procédure de collecte, de traitement de l'information, et de consultation des résultats est en particulier très lourde à gérer quand les effectifs sont importants (Younes, 2006) ;

$>$ L'analyse de la validité et la fiabilité interne de l'instrument de meure mis en place ne se fait pas dans la plupart du temps (outils, méthodes de travail, cohérence et efficacité interne) (Dejean, 2002; Knight \& Trowler, 2001; Van Damme, 2003).

\section{Objectifs et questions de la recherche}

Cette recherche est limitée essentiellement à la conception et à la validation d'un instrument de mesure informatisé d'évaluation des enseignements et des formations par les étudiants au sein de la faculté des sciences Ben M’Sik. Maroc.

De façon plus précise, le présent travail a pour objectif, de répondre aux deux questions suivantes :

$\checkmark$ Comment peut-on élaborer un instrument d'évaluation explicite quant à ses critères, et qui permet de gérer aisément la procédure et la mise en œuvre (administration des questionnaires, de compilation des données, de suivi de l'évaluation) de l'évaluation?

$\checkmark$ Dans quelle mesure l'instrument de mesure d'évaluation élaboré présente-t-il certaines qualités métrologiques ?

Afin de répondre à la problématique, trois objectifs sont fixés :

1- Choisir le modèle d'évaluation sur lequel nous allons nous baser pour élaborer l'instrument ;

2- Informatiser l'instrument de mesure;

3- Evaluer les qualités métrologiques de cet instrument, plus précisément vérifier sa validité interne et sa fidélité.

\footnotetext{
${ }^{5}$ Loi 01-00 Article 79 : Pour la réalisation des audits et de l'évaluation requises à l'article 77 ci dessus, il sera procédé à la création d'instances spécialisées de régulation bénéficiant de l'autonomie et de l'indépendance nécessaires, notamment une instance nationale d'évaluation et un observatoire pour l'adéquation des enseignements supérieurs à l'environnement économique et professionnel.
} 


\section{Conception de l'instrument de mesure}

a. Les critères d'évaluation

o Pour l'évaluation de l'enseignement :

Suite aux travaux de Feldman (1976, 1984), Abrami et d'Appolonia (1990), Centra (1993), Marsh (1983), de Gilles et al. (2006), Romainville (2009), nous retenons comme pertinentes pour l'évaluation de l'enseignement les dimensions suivantes: l'organisation de l'enseignement, la pédagogie, la relation avec les enseignants et les modalités d'évaluation des étudiants.

o Pour l'évaluation de la formation:

L'évaluation de l'organisation de la formation permet de connaître les appréciations des étudiants sur les finalités de la formation, le degré des cohérences horizontales, verticales et d'ensemble, ainsi que l'identification des points forts, faiblesses et dysfonctionnements du système de formation.

Nous avons retenu comme pertinentes pour l'évaluation de la formation les dimensions suivantes: finalités de la formation, organisation des enseignements, acquisitions, emploi du temps, contenu de la formation, organisation matérielle et administrative et la communication au sein de la faculté.

b. L’instrument de l'évaluation : Questionnaire

Les dimensions de l'évaluation et les critères sont reflétés par des items spécifiques dans deux questionnaires de type multidimensionnel. Ils se présentent sous la forme d'une liste d’items spécifiques reflétant les dimensions servant à décrire l'efficacité de l'enseignement et de la formation, et d'items d'appréciation globale. Ces items sont des propositions par rapport auxquelles les étudiants se positionnent sur une échelle de type Likert en 4 points : pas du tout, plutôt non, plutôt oui, tout à fait, en plus une case " sans avis » et un espace pour avis et remarques.

30 questions spécifiques constituent le questionnaire d’évaluation de l'enseignement, plus 5 questions d’ordre général portant sur l'avis des étudiants vis-à-vis de l'enseignement.

Pour le questionnaire d'évaluation de la formation, 23 questions spécifiques sont proposées, plus une question d’ordre général portant sur la satisfaction des étudiants vis-à-vis de la formation.

\section{Informatisation et mise en place de l'instrument de mesure}

c. Objectifs de l'informatisation de l'outil

Les objectifs de l'informatisation de l'instrument sont :

- Réaliser des questionnaires de façon plus commode;

- Diffuser les questionnaires en ligne aux étudiants ; 
- Optimiser la gestion du processus d'évaluation et minimiser les erreurs de traitement ;

- Rendre et obtenir rapidement les résultats de l'évaluation ;

- Gagner du temps pendant la séance du cours par rapport au processus d'administration des questionnaires papier.

d. Choix de réaliser l'évaluation en ligne

Le choix de réaliser l'évaluation en ligne s'est révélé le plus approprié dans la perspective d'un outil d'évaluation léger sur le plan de l'organisation et de la gestion. Utiliser les réseaux permet de mettre immédiatement à disposition les questionnaires et de les gérer .

e. Technologie utilisée

L’outil élaboré a été conçu en utilisant Joomla pour créer le portail d'évaluation et Limesurvy pour créer, gérer et organiser l'évaluation.

f. Fonctionnalités de l'outil d'évaluation

Les principales fonctionnalités de l'outil sont:

- Accès simple et sécurisé aux questionnaires, convivialité et utilisation, fiabilité de l'information, respect de l'anonymat, structure de l'information, et administration facile.

\section{Mise en œuvre de l'outil informatisé}

g. Échantillon et administration

Après la mise en place de l'outil, nous avons testé l'outil auprès de 222 étudiants de différentes filières.

L'administration des questionnaires a été faite en ligne. Nous avons récupéré les emails des étudiants, puis nous avons créé des invitations pour chaque étudiant, ces invitations vont permettre aux étudiants de recevoir leur code d'accès aux questionnaires.

\section{Exemple de résultats de l'évaluation la Filière SVI}

- La dimension « Organisation des enseignements »:

Concernat cette dimension plus de $50 \%$ des étudiants sont satisfaits de leurs enseignements du point de vue de cohérence, de complémentarité et de coordination (voir annexe 1).

- La dimension « Emploi du temps»:

Pour cette dimension, certains aspects sont évalués positivement, par contre, d'autres aspects sont évalués négativement.

Plus de 50\% des étudiants disent que l'emploi du temps est respecté, l'organisation des traveaux dirigés (TD) est adéquate ainsi que le nombre d'heure des traveaux pratiques (TP) est suffisant. Par contre les étudiants évaluent négativement l'emploi du temps de leur formation en général et le temps pour préparer les examens en particulier (voir annexe 2). 


\section{Évaluation des qualités métriques de l'outil}

Le but d'évaluer les qualités métrologiques du deux questionnaires est de vérifier leurs performance comme instrument de mesure à savoir la validité de structure et la consistance interne. L'analyse factorielle exploratoire en composante principale est utilisée pour tester la validité interne de l'outil, en vérifiant sa composition factorielle (ACP) ou les dimensions retenues dans l'instrument de mesure et de sa fiabilité (l'Alpha de Cronbach noté $\alpha$ ), en étudiant la consistance interne des items de l'instrument. Cette analyse a été faite à l'aide du logiciel SPSS.

a- Les résultats de l'analyse en composante principale (Questionnaire d'évaluation des enseignements)

Afin de faciliter la purification des deux questionnaires, ainsi que l'interprétation des résultats, nous avons procédé dans un premier temps à la purification de chaque dimension qui constitue chaque questionnaire, puis à la factorisation de tout le questionnaire.

Nous présentons au tableau suivant les dimensions retenues pour le modèle théorique et les facteurs proposés par l'analyse factorielle. Il est intéressant de constater que l'analyse factorielle a confirmé certaines dimensions (relation avec les enseignants) et a séparé d'autres (mode d'évaluation et résultats d'évaluation).

\begin{tabular}{|c|c|}
\hline Dimensions du modèle théorique & Facteurs retenus par l'ACP \\
\hline Organisation de l'enseignement & Organisation de l'enseignement \\
(13 items); & (9 items); \\
Pédagogie (6 items); & Pédagogie (4 items); \\
Relation avec les enseignants & Relation avec les enseignants \\
(3 items); & (3 items); \\
Evaluation (8 items). & Modes d'évaluation (6 items); \\
& Résultats d'évaluation (2 items). \\
\hline
\end{tabular}

Tableau 1. Dimensions du modèle théorique et facteurs retenus par ACP

Afin de confirmer les propriétés psychométriques du nouveau modèle à cinq dimensions obtenues, nous avons effectué une deuxième ACP pour toutes les dimensions du questionnaire. Cette analyse factorielle vise à vérifier le modèle à cinq dimensions que nous avons obtenu après la purification de chaque dimension du questionnaire. Le nombre de facteurs recherchés est fixé à priori à cinq. Le facteur principal de cette solution explique la plus grande partie de la variance, soit 23,123\%. Les quatre autres facteurs qui résument les 15 items ajoutent, ensemble, 45,653 \% à l'explication, pour une variance totale de $68,776 \%$. La variance expliquée augmente donc substantiellement avec le modèle multidimensionnel.

L'analyse factorielle révèle plusieurs composantes mais seulement cinq ont une valeur propre clairement supérieures à 1 . L'inspection du graphique des valeurs propres ci-dessous, témoigne d'une rupture claire 
après la cinquième composante, ce qui confirme le modèle retenu à cinq dimensions.

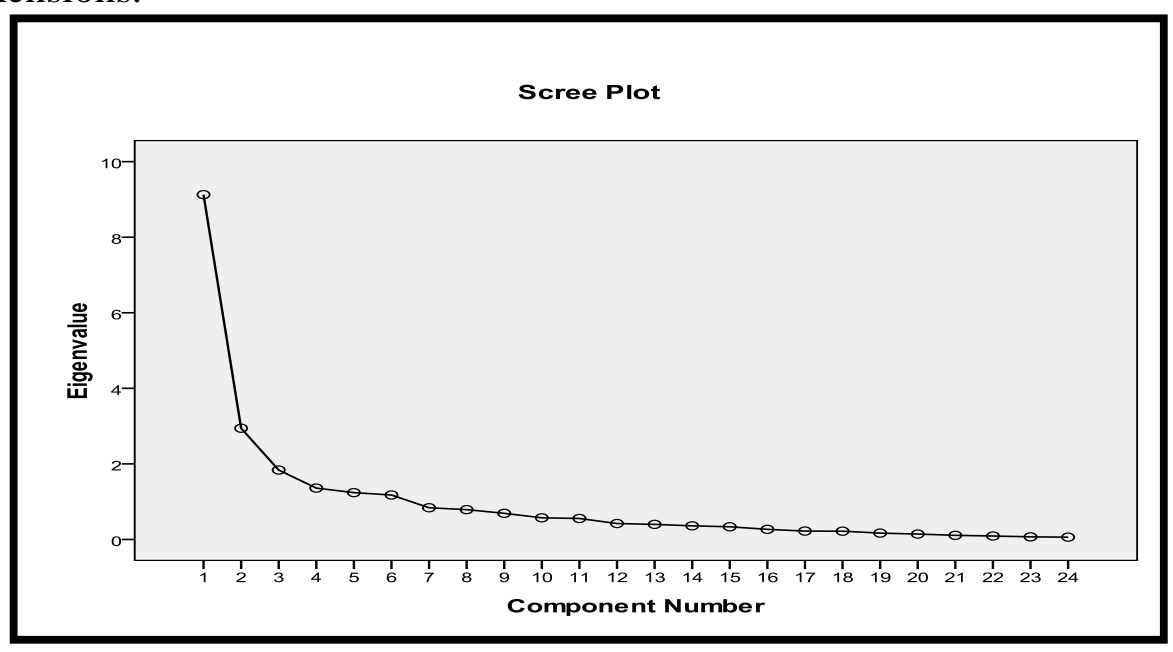

Figure 1. Graphique des valeurs propres

D‘après la structure factorielle obtenue (voir annexe 3 ), la très grande majorité des facteurs sont similaires aux dimensions du modèle obtenu à cinq dimensions. Les énoncés sont associés à leur facteur théorique. La solution factorielle révèle que les cinq facteurs empiriques obtenus correspondent parfaitement aux dimensions théoriques : le facteur 1 (dimension "Organisation de l'enseignement"), le facteur 2 (dimension "relation avec les enseignants"), le facteur 3 (dimension "Modes d’évaluation"), le facteur 4 (dimension "Pédagogie") et le facteur 5 (dimension "résultats d’évaluation").

La plupart des facteurs sont univoques, c'est-à-dire sans double saturation. La matrice des données sont factorisables avec un $\mathrm{KMO}=0,768$ et un Bartlett significatif (Khi deux=778,397, df=276, $\mathrm{p}=0,000$ ).

Les caractéristiques psychométriques du modèle sont satisfaisantes, toutes les communautés étant supérieures à 0.4 et les corrélations des items avec leur facteur étant supérieures à 0.5 .

La fiabilité est vérifiée par l’Alpha de Cronbach qui indique une valeur de 0,922. Les résultats montrent une très bonne homogénéité des items de l'ensemble du questionnaire.

b. Les résultats de l'analyse en composante principale (Questionnaire d’évaluation des formations)

Nous présentons dans le tableau suivant les dimensions retenues pour le modèle théorique et les facteurs proposés par l'analyse factorielle. Il est intéressant de constater que l'analyse factorielle a confirmé certaines dimensions (organisation des enseignements) et a supprimé d’autres (finalité de la formation). 


\begin{tabular}{|c|c|}
\hline Dimensions du modèle théorique & Facteurs retenus par l'ACP \\
\hline $\begin{array}{c}\text { Finalités de la formation (2 items). } \\
\text { Organisation des enseignements (3 items). } \\
\text { Acquisition (2 items). } \\
\text { Emploi du temps (6 items). } \\
\text { Contenu de la formation (2 items). } \\
\text { Organisation matérielle et administrative (5 } \\
\text { items) } \\
\text { La communication au sein de la faculté (3 } \\
\text { items). }\end{array}$ & $\begin{array}{c}\text { Organisation des enseignements } \\
\text { (3 items). } \\
\text { Acquisition (1 items). } \\
\text { Emploi du temps ( } 3 \text { items). } \\
\text { Contenu de la formation (1 items). } \\
\text { Organisation matérielle et administrative } \\
\text { ( } 3 \text { items). } \\
\text { La communication au sein de la faculté (1 } \\
\text { items). }\end{array}$ \\
\hline
\end{tabular}

Tableau 2 Dimensions du modèle théorique et facteurs retenus ACP

Afin de vérifier la structure de ce modèle à six dimensions obtenues, nous avons effectué une deuxième ACP à toutes les dimensions du questionnaire, le but est de vérifier le modèle théorique à 6 dimensions que nous avons obtenu après la purification de chaque dimension du questionnaire. Cette méthode souvent appliquée consiste simplement à fixer a priori le nombre de facteurs selon des critères théoriques, puis à vérifier si la solution, notamment la répartition des items en facteurs, correspond à une solution attendue et interprétable. Le nombre de facteurs recherchés est fixé à priori à six. Le facteur principal de cette solution explique la plus grande partie de la variance, soit 17,635, les cinq autres facteurs qui résument les 9 items ajoutent, ensemble, 63,139 \% à l'explication, pour une variance totale de 80,774. La variance expliquée augmente donc substantiellement avec le modèle multidimensionnel. L'inspection du graphique des valeurs propres ci-dessous, témoigne d'une rupture claire après la sixième composante, ce qui confirme le modèle théorique à six dimensions.

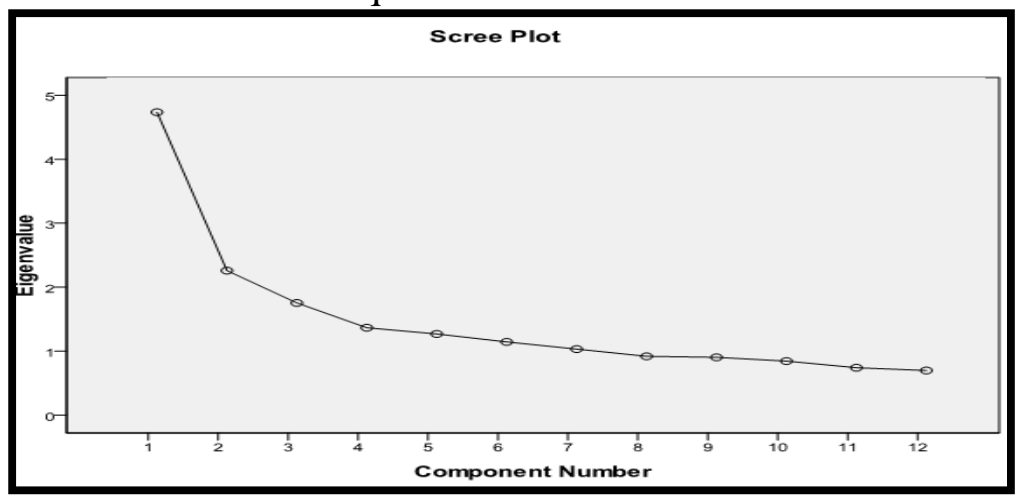

Figure 2. Graphique des valeurs propres

La solution factorielle obtenue (voir annexe 4) révèle que les six facteurs empiriques obtenu correspondent parfaitement aux dimensions théoriques : le facteur 1 (dimension "Organisation des enseignements"), le facteur 2 (dimension "emploi du temps"), le facteur 3 (dimension "contenu de la formation"), le facteur 4 (dimension "Organisation matérielle") et le 
facteur 5(dimension "acquisition"), le facteur 6 (dimension "communication"). La plupart des facteurs sont univoques, c'est-à-dire sans double saturation.

La matrice des données sont factorisables avec un $\mathrm{KMO}=0,765$ et un Bartlett significatif (Khi deux=443,302, df=66, $\mathrm{p}=0,000$ ).

Les indicateurs de structure apparaissent acceptables, la communalité de chaque item est supérieure à 0,4 et les contributions sont tous supérieures à 0,5 . Une analyse de la cohérence interne pour l'ensemble du questionnaire donne une valeur du coefficient alpha est de $a=0,827$. Le degré d'homogénéité est considéré comme très satisfaisant pour les six facteurs selon Robinson, Shaver et Wrightsman (1999).

\section{Conclusion et Perspective}

Le but de cette étude consiste à élaborer et à analyser la structure factorielle d'un instrument de mesure informatisé destiné à évaluer les enseignements et les formations en ligne. L'efficacité et l'utilité de cet outil déclinent en cinq qualités principales: structure de l'information (qualitative et quantitative), fiabilité de la saisie, rapidité des traitements, accessibilité des résultats actualisés, administration et utilisation facile.

L'évaluation des qualités métrologiques de l'instrument a été corroborée par l'analyse factorielle exploratoire ainsi que par la mesure de sa consistance interne. Ces analyses permettent de constater que les deux questionnaires élaborés en l'état actuel pourront être simplifié, en supprimant certains items qui sont jugés par les répondants mal formulés ou bien qui ont la même signification.

Nous pouvons donc conclure que c'est un instrument cohérent du point de vue de mesure et qui peut être utilisé comme outil pour l'évaluation des enseignements et des formations.

\section{References:}

Abrami, P.C., D'apollonia, S. (1990). The Dimensionality of ratings and their use in personnel decisions. In M. Theall \& J. Franklin (Eds.): Students ratings of instruction : Issues for improving practice. New Directions for Teaching and Learning. 43, 97-111.

Bernard, H., \& Bourque, S. (1999). Portrait des politiques et pratiques d'évaluation, d'amélioration et de valorisation de l'enseignement des universités québécoises. Res Acamedica, 1(2), 33-60.

Chemsi, G. (2011). Conception d'un instrument de mesure informatisé d'évaluation des enseignements et des formations par les étudiants : EVALEF (thèse de doctorat). Hassan II University of Casablanca, Maroc.

Centra, J. A. (1993). Reflexive faculty evaluation effectiveness. enhancing teaching and determining faculty effectiveness. San Francisco: Jossey-Bass. 
Cronbach, L.J., Gleser, G.C, Nanda, H. \& Rajaratnam, N. (1972). The dependability of behavioural measurements ; theory of generalizability for scores and profiles. New York : John Wiley.

CSE Maroc. (2008). état et perspectives du système d’éducation et de formation, Rapport analytique, volumes 1, 2, 3 et 4. Savants ayant une connaissance approfondie ;

Dejean, J. (2002). L'évaluation de l'enseignement dans les universités françaises. Paris : Haut Conseil de l'évaluation de l'école, MEN.

Detroz, P. (2010). Le point sur les dispositifs d'évaluation des enseignements par les étudiants : pertinence, utilisation, amélioration. (Thèse de doctorat non publiée). Université de Liege, Liege, Belgique.

Donald, J. \& Saroyan, A. (1991). Assessing the quality of teaching in Canadian University. Commission of inquiry on Canadian university education, Research Report, 3.

De Ketele, J.M. \& Rogiers, X. (1993). Méthodologie du recueil d'information : fondement des méthodes d'observation, de questionnaires, d'intervieus et d'étude d documents. Bruxelles : De Boeck.

Figari, G. (1994). Évaluer: Quel référentiel. Bruxelles: De Boeck Université. Knight, P. \& Trowler, P. R. (2001). Departmental Leadership in Higher Education. Buckingham: SRHE and Open University Press.

Robinson, J. P., Shaver, P. R., \& Wrightman, L. S. (Ed.) (1999). Measures of Personality and Social Psychological Attitudes. États-Unis: Academic Press. Romainville, M. (2009). Une expérience d'élaboration collective de critères de qualité. Dans M. Romainville et C. Coggi (dir.), L'évaluation de l'enseignement par les étudiants. Approches critiques et pratiques innovantes (p.145. 163). Bruxelles : De Boeck.

Van Damme, D. (2003). Standards and indicators in institutional and programme accreditation in higher education. A conceptual framework and a proposal. Paper for the UNESCO-CEPES project. 


\section{Annexe:}

Annexe 1:

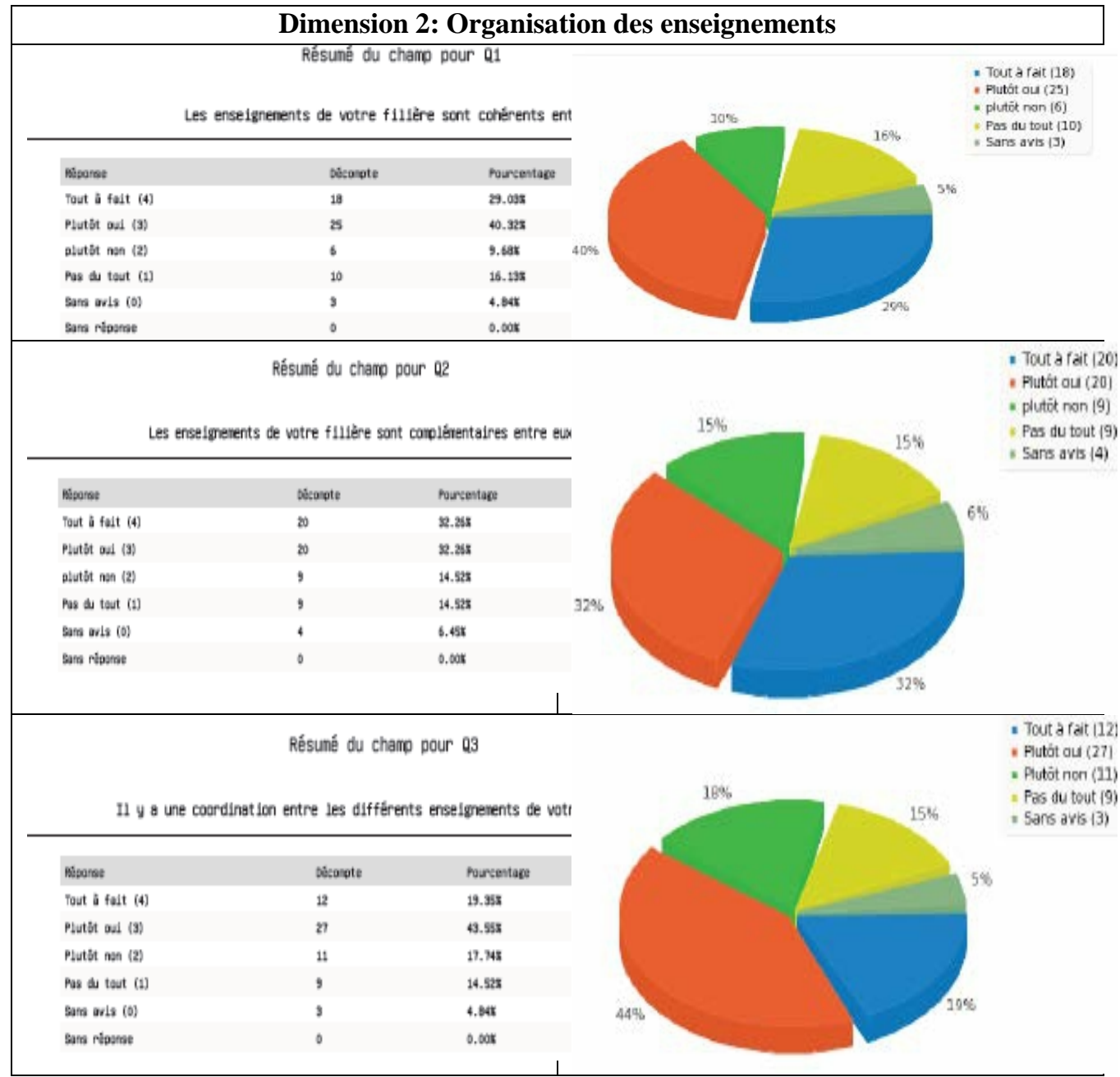


Annexe 2:

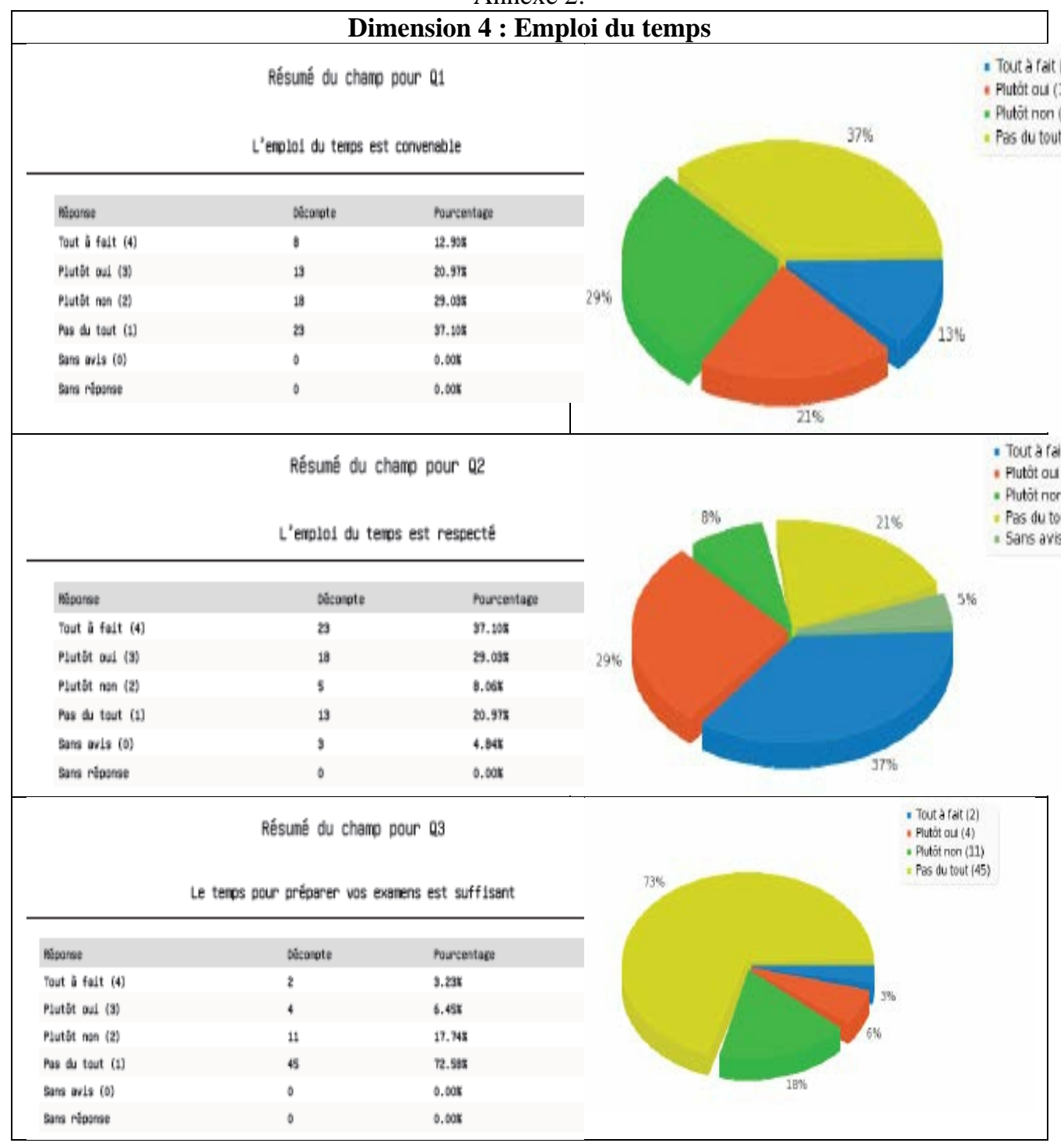


Annexe 3:

\begin{tabular}{|c|c|c|c|c|c|}
\hline \multirow[t]{2}{*}{ Dimensions/items } & \multicolumn{5}{|c|}{ Facteurs } \\
\hline & 1 & 2 & 3 & 4 & 5 \\
\hline $\begin{array}{c}\text { 1) Organisation } \\
\text { Les objectifs/enseignement }\end{array}$ & ,803 & & & & \\
\hline Le contenu/enseignement & ,803 & & & & \\
\hline Le contenu/présenté et discuté à la première séance & ,656 & & & & \\
\hline Le plan/a été respecté & ,751 & & & & \\
\hline Le contenu/structuré & ,818 & & & & \\
\hline Ce cours/acquisition & ,737 & & & & \\
\hline L’unité d'enseignement/adaptable & ,682 & & & & \\
\hline L’horaire prévu/respecté & ,439 & & & ,755 & \\
\hline $\begin{array}{l}\text { La quantité de travail demandé/réaliste adaptable à } \\
\text { votre niveau }\end{array}$ & ,600 & & & & \\
\hline $\begin{array}{l}\text { 2) Pédagogie } \\
\text { Les supports/ compréhension du cours }\end{array}$ & & & & ,776 & \\
\hline Les explications/claires pour vous & & & & ,539 & \\
\hline Le rythme de l'enseignement/adapté à votre niveau & & & & ,438 & \\
\hline $\begin{array}{l}\text { Les enseignements/liens entre les concepts et leurs } \\
\text { applications. }\end{array}$ & & ,614 & & & \\
\hline $\begin{array}{c}\text { 3) Relation/enseignant } \\
\text { L'enseignant(e)/disponible pour les étudiants }\end{array}$ & & ,834 & & & \\
\hline L’enseignant(e)/respect & & ,640 & & & \\
\hline $\begin{array}{c}\text { L'enseignant(e)/respecte les interventions des } \\
\text { étudiants. }\end{array}$ & & ,679 & & & \\
\hline $\begin{array}{l}\text { 4) Les modes d'évaluation } \\
\text { Les modes d'évaluation/formulés clairement. }\end{array}$ & ,539 & & ,549 & & \\
\hline $\begin{array}{l}\text { Les modes d'évaluation/ favorisent l'atteinte des } \\
\text { objectifs du cours. }\end{array}$ & & & ,812 & & \\
\hline $\begin{array}{l}\text { Les évaluations/ sont bien réparties tout au long du } \\
\text { semestre. }\end{array}$ & & & ,700 & & \\
\hline Le nombre de CC/vous semble suffisant. & & & ,728 & & \\
\hline Les critères de correction/sont connus. & & & ,654 & & \\
\hline $\begin{array}{l}\text { Les évaluations/permis de progresser dans votre } \\
\text { apprentissage }\end{array}$ & & $\begin{array}{l}790 \\
\end{array}$ & & & \\
\hline $\begin{array}{l}\text { 5) Résultats d'évaluation } \\
\text { Les notes obtenues/ correspondent au travail que } \\
\text { vous avez fourni. }\end{array}$ & & & & & ,798 \\
\hline $\begin{array}{l}\text { Les résultats des contrôles/communiqués } \\
\text { rapidement. }\end{array}$ & & & & & ,888 \\
\hline
\end{tabular}

Tableau 1: Saturations des items sur chacun des facteurs 
Annexe 4:

\begin{tabular}{|c|c|c|c|c|c|c|}
\hline \multirow{2}{*}{ Dimensions } & \multicolumn{6}{|c|}{ Facteurs } \\
\hline & 1 & 2 & 3 & 4 & 5 & 6 \\
\hline $\begin{array}{l}\text { 1)-Organisation des enseignements } \\
\text { Les enseignements/cohérents entre eux }\end{array}$ & ,888 & & & & & \\
\hline $\begin{array}{l}\text { Les enseignements/complémentaires entre } \\
\text { eux }\end{array}$ & ,875 & & & & & \\
\hline $\begin{array}{l}\text { coordination/enseignements de votre } \\
\text { formation }\end{array}$ & ,636 & & & & & \\
\hline $\begin{array}{l}\text { 2)-Acquisition } \\
\text { Le programme de la formation/acquisition }\end{array}$ & & & & & ,915 & \\
\hline $\begin{array}{l}\text { 3) -Emploi du temps } \\
\text { L'emploi/convenable }\end{array}$ & & ,586 & & & & \\
\hline Le temps/préparation & & ,904 & & & & \\
\hline le nombre d'heures/ examens est suffisant & & ,725 & & & & \\
\hline $\begin{array}{l}\text { 4)-contenu de la formation } \\
\text { le contenu de la formation/structuré }\end{array}$ & & & ,782 & & & \\
\hline $\begin{array}{l}\text { 5)-Organisation matérielle } \\
\text { satisfaction moyens matériels }\end{array}$ & & & & ,803 & & \\
\hline Le matériel dansTP/ suffisant & & & & ,865 & & \\
\hline L'effectif des étudiants en TD/convenable. & & & & ,796 & & \\
\hline $\begin{array}{c}\text { 6)-Communication } \\
\text { L'opération d'information/ orientation }\end{array}$ & & & & & & ,864 \\
\hline
\end{tabular}

Tableau 2. Saturation des items sur chacun des facteurs 